\title{
Measurement of the Proton Moment in Absolute Units
}

\author{
By Harold A. Thomas, Raymond L. Driscoll, and John A. Hipple
}

\begin{abstract}
By measuring the absolute value of the magnetic field and the frequency required for nuclear resonance absorption in a water sample, the gyromagnetic ratio of the proton has been determined to be $\gamma_{p}=(2.67528 \pm 0.00006) \times 10^{4} \mathrm{sec}^{-1}$ gauss ${ }^{-1}$. Using this value with Planck's constant, the value of the magnetic moment of the proton in absolute units becomes $\mu_{p}=(1.4100 \pm 0.0002) \times 10^{-23}$ dyne cm/gauss.

A combination of our result with recent measurements of the proton moment in Bohr magnetons gives a value of $e / m=(1.75890 \pm 0.00005) \times 10^{7} \mathrm{emu}_{\mathrm{gram}}-1$.
\end{abstract}

\section{Introduction}

Since the development of molecular beam [1], ${ }^{1}$ nuclear induction [2], and nuclear resonance absorption [3, 4] techniques, considerable work has been done on the determination of nuclear gyromagnetic ratios. From this ratio and the nuclear spin, the magnetic moment of the nucleus can be calculated.

The measurement of a gyromagnetic ratio $\gamma$ involves the measurement of the frequency, $\nu$, and magnetic field of induction $B_{0}$ required for resonance as indicated by the condition for resonance, $\omega=2 \pi \nu=\gamma B_{0}$. The comparison of either gyromagnetic ratios or magnetic moments requires only frequency determinations, and for this reason much of the data on magnetic moments now available is of this type. A few direct measurements of gyromagnetic ratios in absolute units have been made with accuracies of the order of 0.5 percent, which is about the best that can be done with the ordinary techniques of measuring magnetic fields. In the experiment reported here, the proton gyromagnetic ratio has been determined with much greater accuracy by using more elaborate methods of measuring the magnetic field and frequency. This precise measurement will allow previous relative determinations to be recalculated in absolute units, and will also provide a convenient standard of magnetic field for the measurement of other atomic constants.

\footnotetext{
1 Figures in brackets indicate the literature references at the end of this paper.
}

The nuclear absorption method of Purcell $[3,4]$ was used for detecting resonance, because the field involved lends itself more readily to precise measurement than that used in the molecular beam method, and the apparatus appeared somewhat simpler to construct than that employed by Bloch in the nuclear induction experiment.

The use of a solenoid of known dimensions would provide the most accurately known magnetic field, but this possibility was discarded, because the maximum field available would only be of the order of 20 gausses. The signal-to-noise ratio becomes very low in such a weak field, and at the time this experiment was planned no attempt had yet been made to work in this range. ${ }^{2}$ An iron-cored electromagnet with a relatively strong field was used, which reduced the signalto-noise problem but required auxiliary apparatus for measuring the field.

To measure the field, a Pellat electrodynamometer [6] could be used but would require a large gap and a correspondingly large magnet. The method finally chosen is one similar to that previously described in the literature $[7,8,9]$ for measuring magnetic fields. In this method one measures the force on a known length of wire carrying a known current in the magnetic field to be determined. A long rectangular coil is suspended vertically from an analytical balance

2 The recent success of Brown and Purcell [5] in working in fields as low as 11 gausses now makes the solenoid method appear more attractive than when the present work was undertaken; as a means of eliminating systematic errors and possibly improving the accuracy, it is important that the measurements described here be repeated with this method. This is now being done. 
with the lower end in the gap of the magnet. The vertical sides of the coil act as the connecting leads to the "force conductors", which are formed by the lower horizontal portion of the coil. The fringing field at the upper end of the coil may be reduced to a negligible value by means of Helmholtz coils. Figure 1 is a photograph of the over-all experimental setup showing the magnet, coil, balance, current measuring apparatus, and

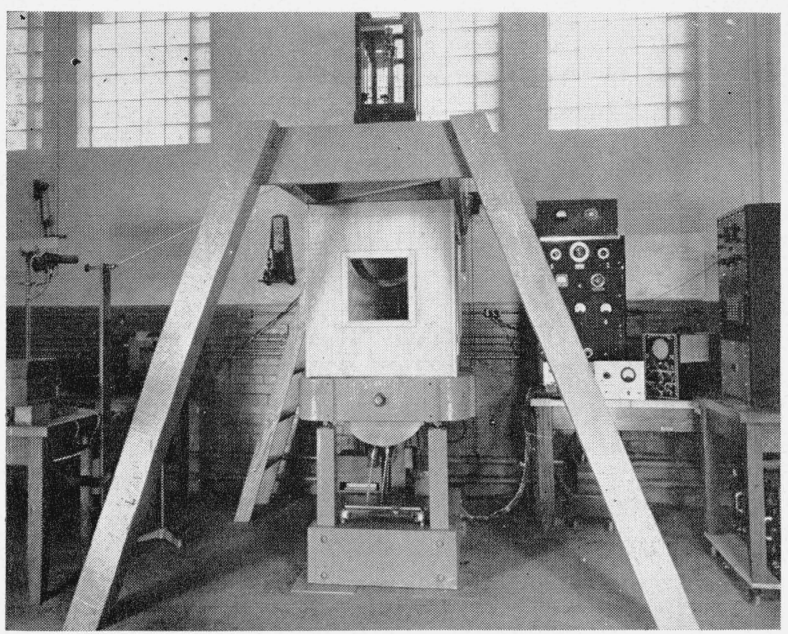

Figure 1. General view of magnet, balance, and proton resonance equipment.

electronic equipment for regulating the magnet and detecting the resonance absorption.

Since it is the average field over the region occupied by the force conductors that is measured by the force method, it is essential that the field distribution be precisely determined, and that the field in this region be reasonably uniform. Then, from the value of the average field and the field distribution, one can determine the value of the field at the proton sample, which may be located just below the coil.

The value of the field was adjusted so that the resonance frequency would be $20 \mathrm{Mc}$, which can be heterodyned directly with the Bureau's radio station WWV for the frequency measurement.

\section{Magnet Design}

Since the method of field measurement employed requires a homogeneous field of considerable extent, quite large pole faces are required. This limits the maximum field intensity available if the magnet is to be held to a reasonable size and cost. For proton resonance at $20 \mathrm{Mc}$, the maxi- mum field must be about 5,000 gausses. The coil, being suspended from a balance, is quite sensitive to air currents, and for this reason particular care was used in the design of the magnet to insure that all exposed surfaces of the windings and pole face would be sufficiently close to room temperature to minimize the effect of thermal air currents. These considerations led to a magnet design shown in some detail in figure 2 .

Performance tests showed the magnet to operate satisfactorily up to 5,000 gausses; however, beyond this point the leakage flux from the sides of the poles to the yoke was great enough to produce partial saturation of the yoke. At 5,000 gausses the magnet requires $6.7 \mathrm{amp}$ at $150 \mathrm{v}$, and the temperature rise in the hottest point of the windings is $30^{\circ} \mathrm{C}$ with a water flow of 1.6 liters a minute. The time constant of the magnet is 2.7 sec.

While the yoke is operating near saturation at the maximum value of field, the usable portion of the pole faces is operating at only about 5,000 gausses, which is sufficiently low to insure a stable field distribution.

\section{Nuclear Resonance Techniques}

For the detection of the proton resonance absorption, a radio frequer cy bridge similar to that used by Purcell and his collaborators [4] was constructed. This arrangement, however, had two serious drawbacks for this application. It was quite sensitive to microphonics so that it could not be easily used to probe the field for determining its distribution; and, since it shows the dispersion as well as the absorption, there would be greater uncertainty involved in determining the exact center of resonance. For these reasons, this method was finally rejected and a new amplitude bridge arrangement for detecting nuclear resonance was developed [10]. As its name implies, this bridge detects only amplitude changes in the radio-frequency voltage across the absorption coil and does not respond to phase changes. As a result, response to microphonic disturbances, which are largely reactive, is reduced by several orders of magnitude. Similarly, the reactive or dispersion component of the nuclear absorption signal is reduced to a negligible second-order correction, and only the resistive or absorption component is detected. 

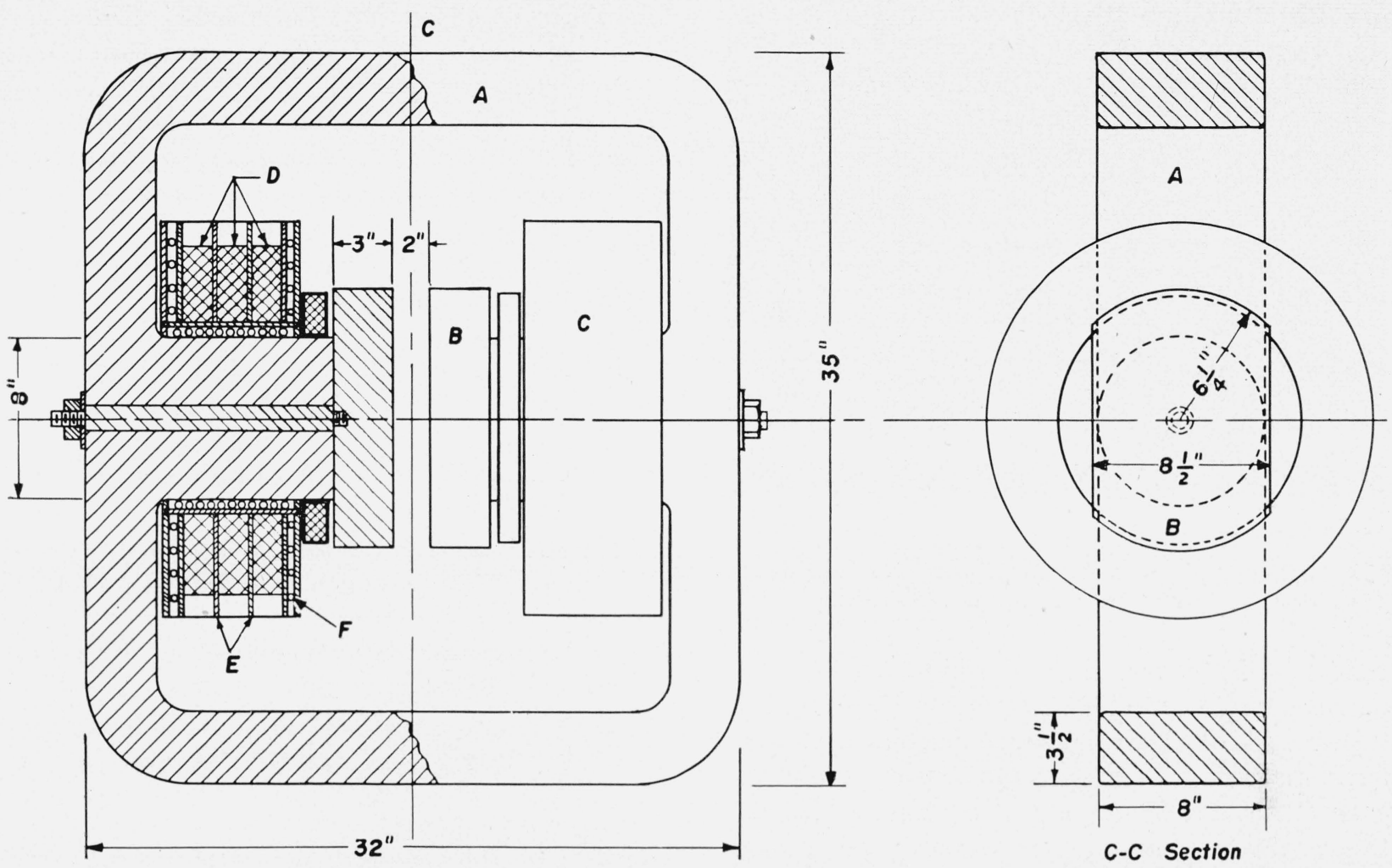

Figure 2. Magnet design details.

Figure 3 shows the essential details of an amplitude bridge for operating at $20 \mathrm{Mc}$. The proton sample is placed in $L_{1}$, which is tuned to resonance by $C_{1}$ and fed from a radio-frequency generator through a resistance, $R_{1}$, much higher than the

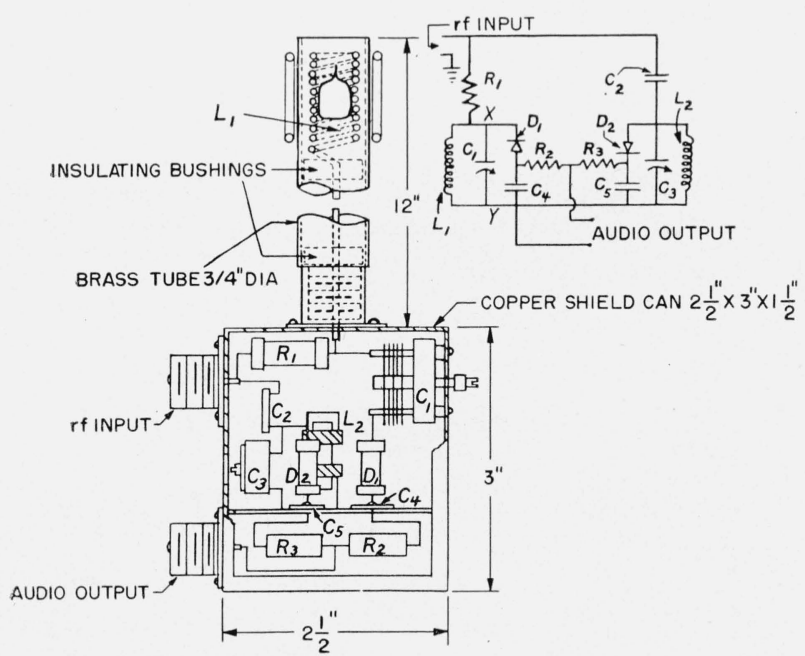

Figure 3. Amplitude bridge for detecting nuclear resonance.

$\mathrm{R}_{1}=25,000$ ohms; $\mathrm{R}_{2}, \mathrm{R}_{3}=50,000$ ohms; $\mathrm{C}_{1}=120 \mathrm{mmf} ; \mathrm{C}_{2}=2 \mathrm{mmf}$ mica; $\mathrm{C}_{3}=$ $7 \mathrm{mmf}$ ceramic trimmer; $\mathrm{C}_{4}, \mathrm{C}_{5}=500 \mathrm{mmf}$ feed through condenser; $\mathrm{L}_{2}=1$ millihenrie r-f choke; $D_{1}, D_{2}=1$ N34 crystal diodes.

Measurement of the Proton Moment in Absolute Units resonant impedance of $L_{1}-C_{1}$. The field is modulated locally a few tenths of a gauss by applying a $20-\mathrm{c} / \mathrm{s}$ voliage to the small Helmholtz coils located on either side of the sample. As the magnetic field passes through the value required for nuclear resonance, the nuclear absorption lowers the $Q$, causing the voltage across $L_{1}$ to change. This change in the amplitude of the radio-frequency voltage is detected by a diode detector, $D_{1}$, and the resulting audio resonance signal can be amplified by a highgain audio amplifier and displayed on an oscilloscope. To eliminate noise and hum modulation present in the radio-frequency source, a capacitance voltage divider and diode rectifying in the reverse phase to $D_{1}$ are used and the outputs combined in an audio bridge network. Audio modulation in the radio frequency is thus balanced out, but the nuclear absorption signal, detected by only one diode, appears at the output reduced by a factor of two. Sufficient cancellation of the noise modulation is usually obtained by merely adjusting $C_{3}$.

The signal-to-noise ratio obtainable with this circuit depends largely on the type of diodes used, approaching the theoretical maximum for pure 


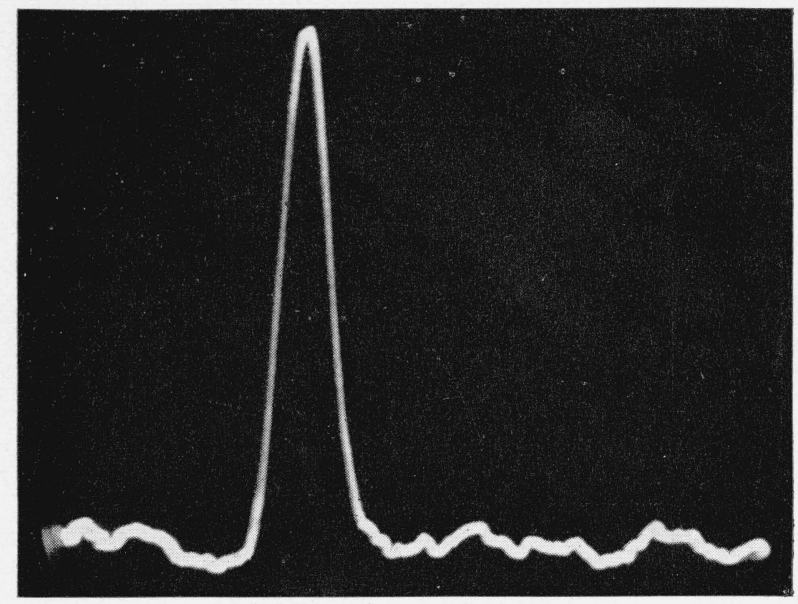

Figure 4. Proton resonance line detected with amplitude bridge using crystal detectors.

The field intensity was 4,700 gausses, and line width is 0.3 gauss.

tungsten filament diodes, and being much lower for oxide-coated cathode or crystal diodes. The flicker noise of the oxide-coated diode is quite similar in magnitude and frequency distribution to the contact noise of the crystal diode, and at the low frequencies it predominates over shot and thermal noise. In spite of the higher noise figure, the crystal diodes work quite satisfactorily for detecting the relatively strong proton signal at fields above a few thousand gausses and were used exclusively throughout this experiment because of the circuit simplicity gained by their use. Figure 4 shows a typical proton resonance line using a water sample with the relaxation time [2] $T_{1}$ adjusted with a dilute solution of ferric nitrate [4]. The line width shown in figure 4 is 0.3 gauss, and the field is 4,700 gausses. During the experiment the line width was somewhat less than this. A comparison of the sample used with oil samples containing no ferric nitrate indicated that the change in susceptibility of the sample due to the ferric salt was not detectable and could not have caused an error of more than 4 parts per million in the final result.

\section{Magnet Regulator Employing Proton Resonance}

Stabilization of the magnetic field was accomplished by using the signal from a resonance detector in a feedback circuit, as shown in figure 5. If the field at the sample is modulated by an amount considerably less than the resonance line width as shown, the output signal is essentially sinusoidal, and its phase and amplitude depend upon the value of the steady field as indicated. After amplification in a narrow-band amplifier, the sinusoidal voltage from the resonance detector is mixed in the phase detector with a signal from the oscillator supplying the coils modulating the magnetic field. The output of the phase detector is the d-c voltage shown in figure 5, which goes through zero as $B$ passes through the value required for the center of the resonance line. This $\mathrm{d}-\mathrm{c}$ voltage is applied to a power amplifier that excites the auxiliary regulating coils on the magnet. Hunting was eliminated by adding a large $\mathrm{RC}$ time constant to the output of "the phase detector. The main exciting coils were energized by storage batteries. Because of the large time constant of the magnet $(2.7 \mathrm{sec})$, it is not necessary for the regulator to compensate for rapid fluctuations of exciter current.

The resonance detector used is the same as shown in figure 3 , and again water was used for the sample. Both the resonance probe for displaying the resonance line on the oscilloscope and the one for the regulator were excited from the same 20-Mc crystal oscillator. The frequency from this crystal oscillator varied less than 1 part per million (ppm) after a $3-\mathrm{hr}$ warm-up period, as shown in figure 6. This curve was checked a number of times by heterodyning with WWV and measuring the difference frequency.

Figure 7 shows the circuit details of the magnet regulator. By observing the shift in the proton line from one probe, it was found that the regulator reduced the effect of a 2-percent change in

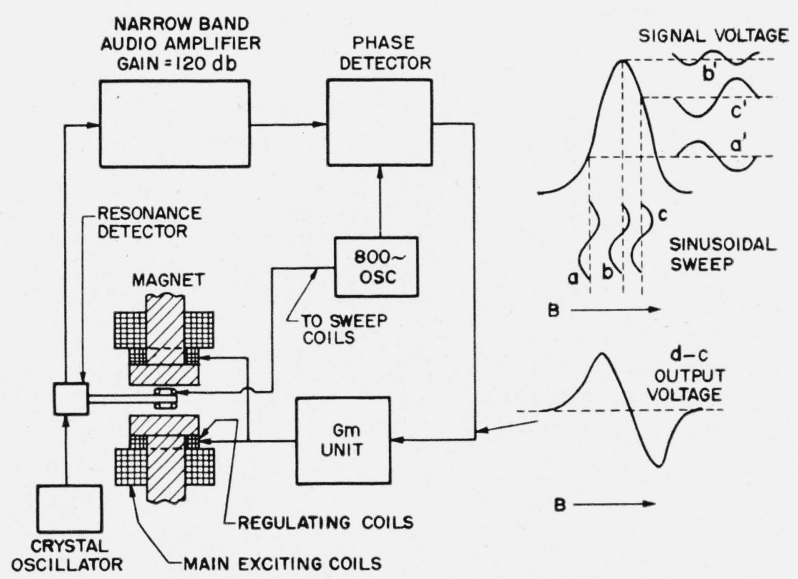

Figure 5. Proton-resonance magnet regulator 
main exciting current to 0.02 gauss in 4,700 gausses.

\section{Field Distribution}

To shim the magnetic field and determine the field distribution, one resonance probe was used to regulate the magnetic field, and another resonance probe was mounted on a movable carriage so that it could be moved easily in the magnetic field while watching the proton pip. As the field does not vary with time, variations in the field as a function of position could be observed as a shift in the position of the proton pip on the oscilloscope

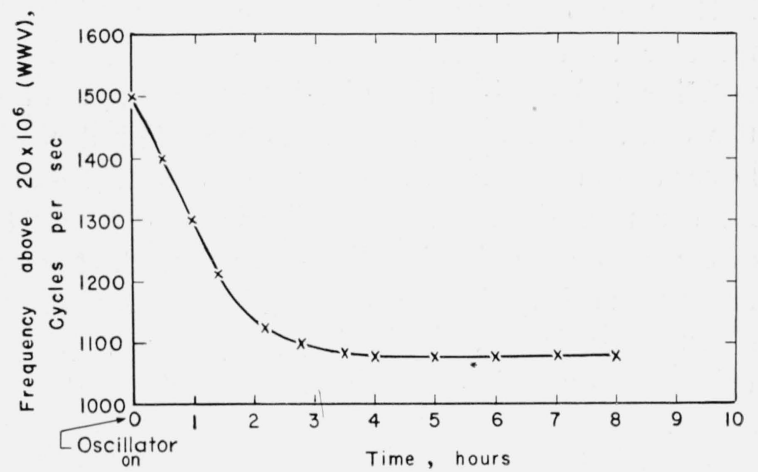

Figure 6. Stability of crystal oscillator. screen. The oscilloscope sweep voltage was obtained from the sinusoidal voltage applied to the field sweep coils located on either side of the sample (see fig. 3). This voltage was also put through a peak-clipping and differentiating circuit, and the resulting pulses mixed with the resonance signal so that vertical marking lines would also appear on the scope. The shift of the resonance pip with change in field could then be observed relative to these markers with good precision.

In order to measure the distribution, another pair of small Helmholtz coils were mounted on the movable probe on either side of the sample, as shown in the photograph of figure 8. These Helmholtz coils were calibrated by changing the radio frequency applied to the probe by a small known amount that would shift the resonance pip off the marker line. The pip was then brought back into the original position by sending a small current through the Helmholtz coils. Then from $\Delta \omega=\gamma \Delta B$ (where $\gamma$ is known to better than $1 \%$ ) the Helmholtz coil constant was calculated. Ten determinations of the coil constant by this method showed a maximum deviation of 3 percent. The method used to measure the frequency change introduced an error mainly due to the uncertainty

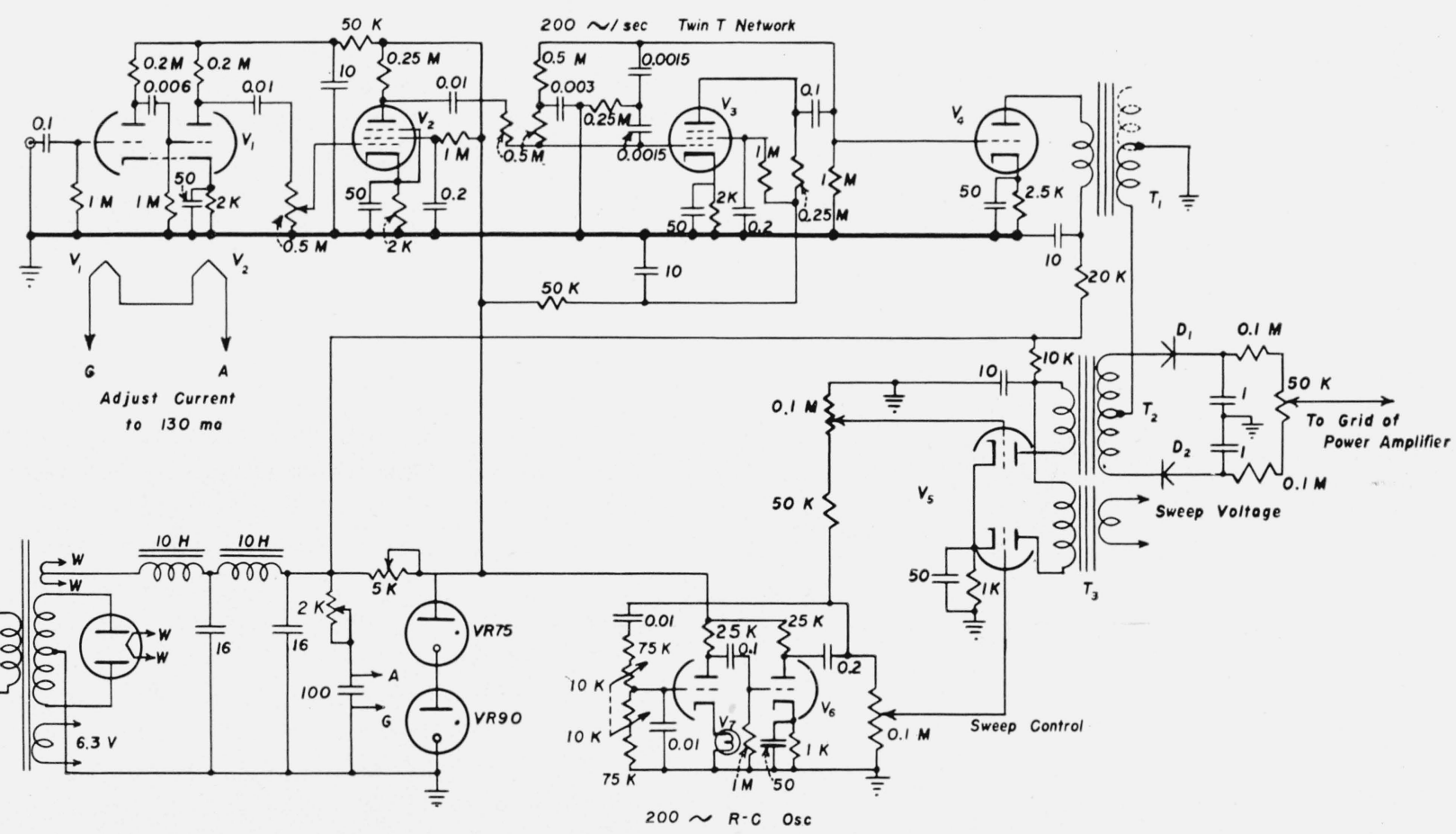

Figure 7. Circuit details of magnet regulator.

$\mathrm{V}_{1}, 12 \mathrm{SC} 7 ; \mathrm{V}_{2}, 12 \mathrm{SJ} 7 ; \mathrm{V}_{3}, 6 \mathrm{SJ} 7 ; \mathrm{V}_{4}, 6 \mathrm{~J} 5 ; \mathrm{V}_{5}, 6 \mathrm{SN} 7 ; \mathrm{V}_{6}, 6 \mathrm{SN} 7 ; \mathrm{V}_{7}, 4$-watt 120-volt lamp; $\mathrm{D}_{1}=\mathrm{D}_{2}, \mathrm{NN}_{34}$ crystal diodes; T $=\mathrm{T}_{2}$, thordarson $\mathrm{T}-20 \mathrm{D}_{7}$; $\mathrm{T}_{3}$, universal output transformer; capacitances are in $\mu \mathrm{f}$; $\mathrm{K}$, thousands of ohms; $\mathrm{M}$, megohms. 


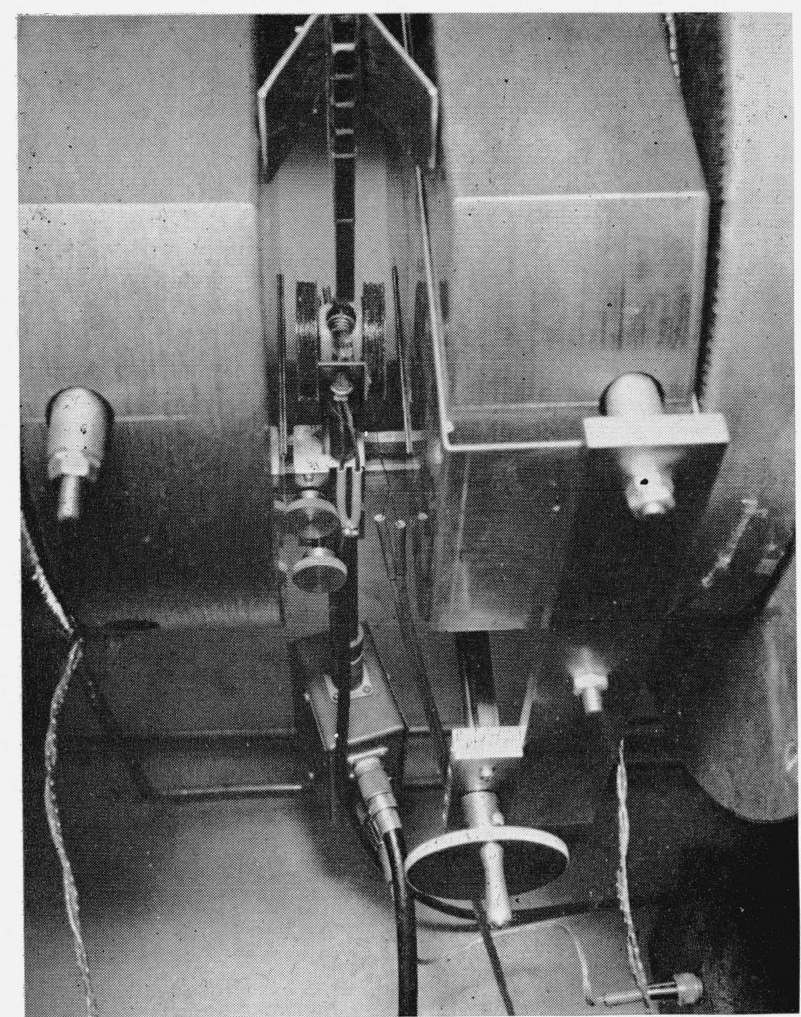

Figure 8. Movable resonance probe.

in reading a calibrated audio oscillator that was estimated at 2 percent. The coil constant for this method of calibration was then taken as $[0.055 \pm$ $5 \%$ ] gauss/ma.

The gap was shimmed by using 2-mil nickel sheets fastened to a brass plate clamped to one of the pole faces. As thinner shims were not immediately available, the gap initially was shimmed on only one side. This gave rise to a slight gradient in the direction of the field, as may

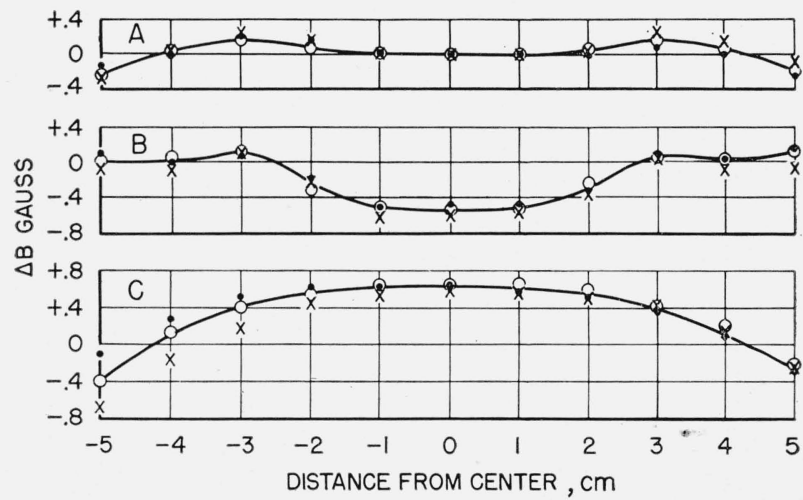

FIGURE 9. Field distribution.

$\mathrm{A}$, at center of gap; B, $+0.5 \mathrm{~cm}$ off gap center; $\mathrm{C},-0.5 \mathrm{~cm}$ off gap center. $\mathrm{O}, 10 / 21 / 48 ; \times, 11 / 16 / 48 ; \bullet, 3 / 4 / 49$. be seen by the field distribution curves shown in figure 9. Complete field plots were made on three different dates as indicated: before, during, and after the set of measurements were taken from which the gyromagnetic ratio was determined. In addition, frequent spot checks were made on the field distribution during the period when the absolute value of the field required for resonance was being obtained. The stability of the field distribution appeared very good, and no variation greater than that indicated in figure 9 was found. From the curves of figure 9, the average field over the region occupied by the bottom conductors of the force coil was found relative to the value where the nuclear sample was located during the absolute measurement of the field. The average field $\overline{\mathrm{B}}$ was found to be lower than the field at the sample $B_{0}$ by 0.04 gauss. As the reproducibility of adjusting to the center of the resonance line is a little less than 0.02 gauss, the uncertainty in the calibration of the Helmholtz coils is negligible in comparison. Hence this field correction may be taken as $(0.04 \pm 0.02)$ gauss.

The deviation of the magnetic field from horizontal direction could contribute some error, but this was found to be less than $1 \mathrm{ppm}$. It was experimentally determined that a lack of perfect alinement of the plane of the rectangular coil in the gap, that is, a slight rotation about its point of support, would also have a negligible effect.

\section{Theory of the Magnetic Balance}

The general method employed in the measurement of the magnetic field has already been described briefly. A period of about $30 \mathrm{~min}$ is required to make a determination. The full accuracy of the method cannot be realized unless the field is held constant during this period. It has been shown elsewhere in this paper how the effect of nuclear resonance absorption itself has been used to satisfy this condition to a high degree of perfection.

In principle, the method is based on the fact that an element of conductor of length $d l$ cm carrying current $i \mathrm{cgs}$ emu perpendicular to a magnetic field of induction $B$ gauss experiences a force $d F$ dynes directed at right angles both to itself and to the field that is given by the following relation,

$$
d F=i B d l \text {. }
$$


As the force is measured in the vertical or $y$ direction, the component of $d l$ in the horizontal or $x$ direction, $d x$, produces a force at the knife-edge of the balance. In order to arrive at the total force produced on a complete coil, it is necessary to integrate eq 1 over all its elements, as indicated below.

$$
F=\int_{C o i l} i B(x, y) d x \text {. }
$$

The current, $i$, being everywhere the same in the circuit, the integration can be carried out numerically from the results of measurements on the width of the coil at various vertical or $y$ positions, together with a measure of the field $B(x, y)$ at these vertical positions.

We may define the effective width of this coil when used as described with this particular magnet by the relationship

$$
X=\frac{1}{\bar{B}} \int_{\text {Coil }} B(x, y) d x .
$$

The value of $X$ thus defined would be considerably affected by the stray field at the top wires of the coil; however, this field has been neutralized by the large Helmholtz coils described previously. The observed force $F$ (dynes) in terms of $\bar{B}, X$, and $I$ (amperes) in a coil of $n$ turns is

$$
F=\frac{n I X}{10} \bar{B}
$$

From this the value of the average field is

$$
\bar{B}=\frac{10 F}{n I X}
$$

\section{Construction of the Coil}

A photograph of the completed coil is shown in figure 10. The nominal dimensions of the plate glass form are shown in figure 11 . The coil has nine turns of wire that lie in grooves formed around the edge of the plate. The wires pass around the sides and bottom of the plate parallel to the edge of the glass and cross over to the next groove at the top. The horizontal stripes shown are silk ties that bind the wires and prevent accidental lateral displacement. The method of forming the grooves on the edges of the glass was similar to that devised by Moon [11]. The manner of supporting the form during lapping is shown in figure 12 .

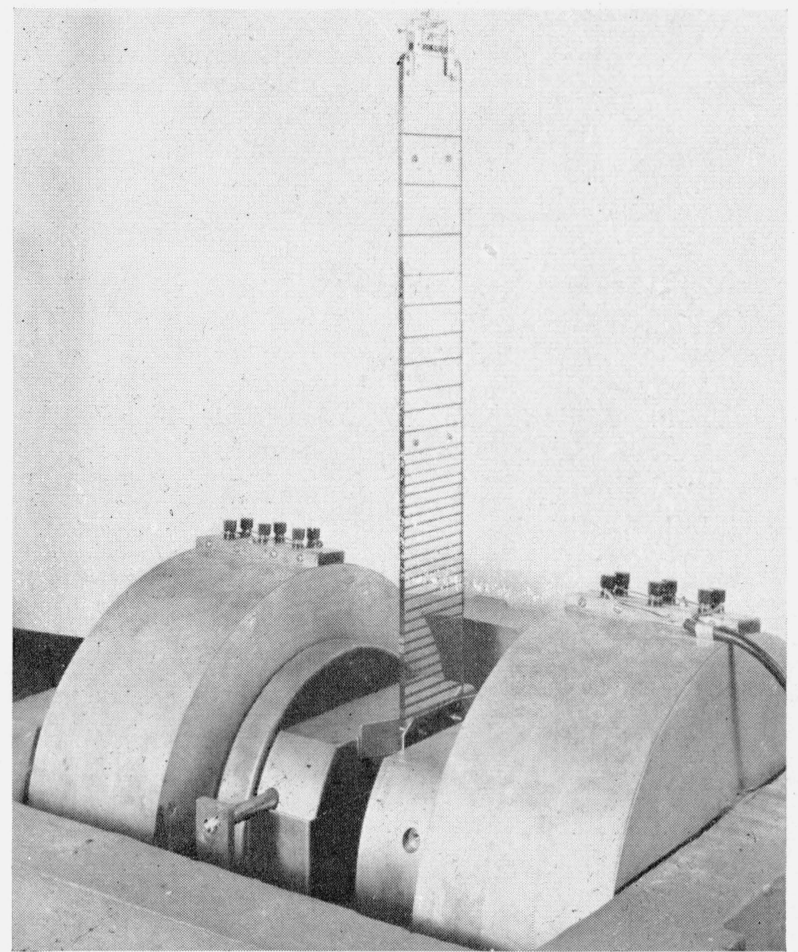

Figure 10. Completed coil suspended in the magnet gap.

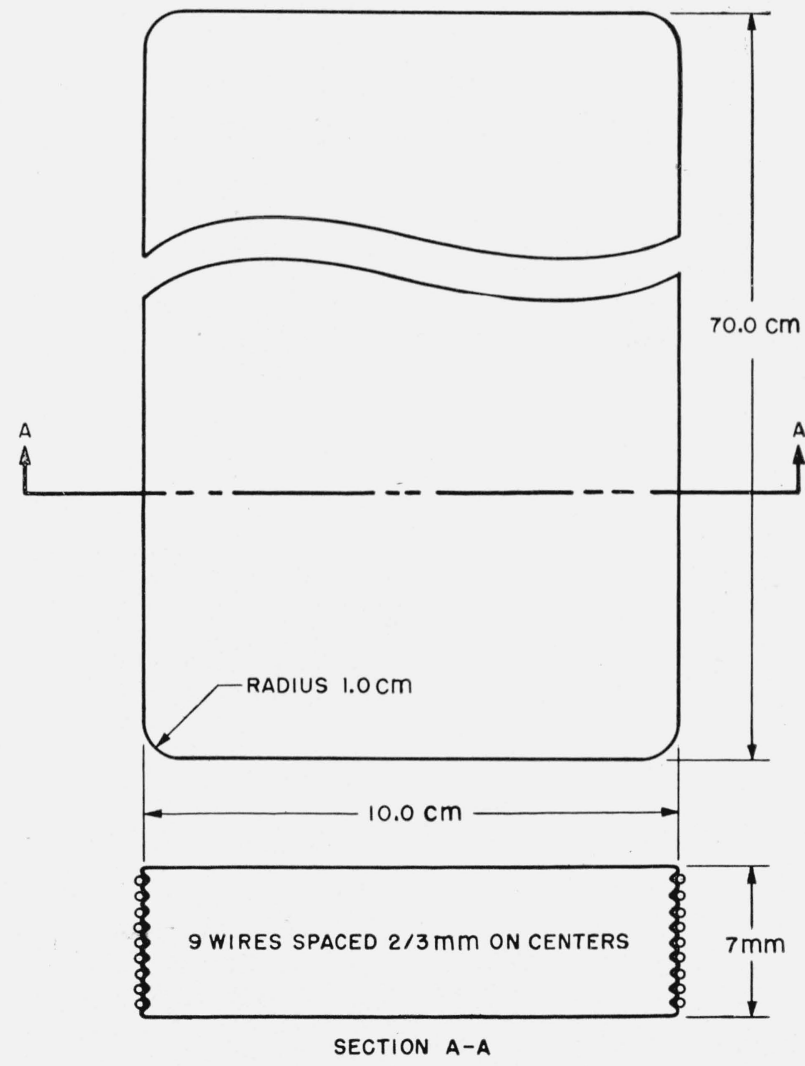

Figure 11. Coil form. 


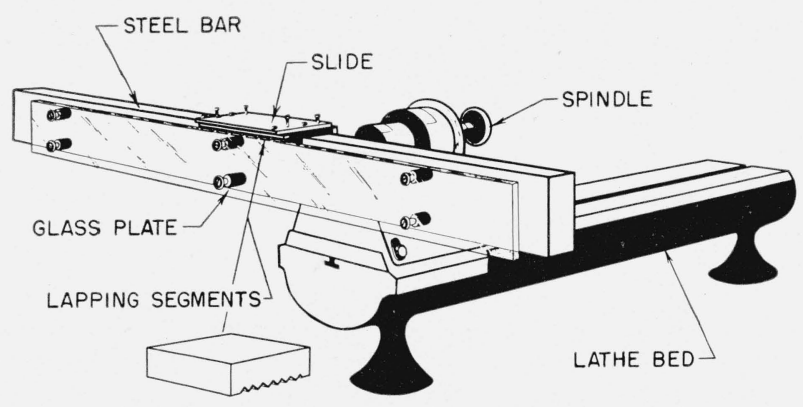

FIGURE 12. Method of lapping grooves on edge of glass plate.

The steel bar to which the plate is secured was annealed and ground parallel on the opposite faces. In addition to holding the glass, the edges of the bar were used to restrain the motion of the lapping tool to a straight line. The depth of lapping was controlled by adjusting the height of the lap relative to the edge of the bar. The assembly was rotated for alternate lapping on the two sides of the form. This procedure assured the same form of grooves on both sides. Grooves were worked around the corners and bottom by hand, using the flat side of the steel bar to keep the grooves in alinement. On completion, the over-all width of the coil form was practically as uniform as that of the bar.

It was convenient to place the wire on the form while the latter was securely fastened to the steel bar. Copper wire of the oxygen-free variety was drawn down through a number of dies from $1 \mathrm{~mm}$ to about $0.56 \mathrm{~mm}$. The last drawing was carried out in a hallway in one straight piece and was wound directly onto the form without coming into contact with anything but the glass. A steady tension of $5 \mathrm{lb}$ was held on the wire during winding. Current leads are brought out centrally at the top of the coil, and potential leads are fastened at the extreme ends of the winding near the edges of the plate.

\section{Measurement of the Coil}

Two measurements are necessary to get the distance between the central filaments of the wires: the over-all width of the coil and the mean diameter of the wire.

The coil was arranged for measurement in a temperature-controlled cabinet, as shown in figure 13. In this setup the width of each turn could be measured at various vertical positions. Measurements were made relative to Johannson gage blocks by means of a motor-driven micrometer devised by Moon [12]. This micrometer was provided with gear to bring it into position for each reading without having to enter the cabinet. Provisions were made for all essential adjustments of micrometer, coil, and end standard from the outside. Readings of the micrometer were estimated to $0.1 \mu$ and would repeat to about $0.2 \mu$. The temperature of the end standard was ascertained from readings taken on an attached thermocouple, and that of the coil was gathered from thermometers plastered to it in several places. Most of the readings were taken at a nominal temperature of $25^{\circ} \mathrm{C}$, but the coil was measured in several places at $29^{\circ}$ and $33^{\circ} \mathrm{C}$, making it possible to correct for the effect of temperature on the width.

The resistance of the winding was established at a known temperature. This was of use later when making corrections to the force on account of the expansion of the coil. The resistance of the coil was used as a measure of its temperature.

Results of measurements on the coil are shown in table 1, a. The result shown for each vertical position is the mean of the nine wires. Measure-

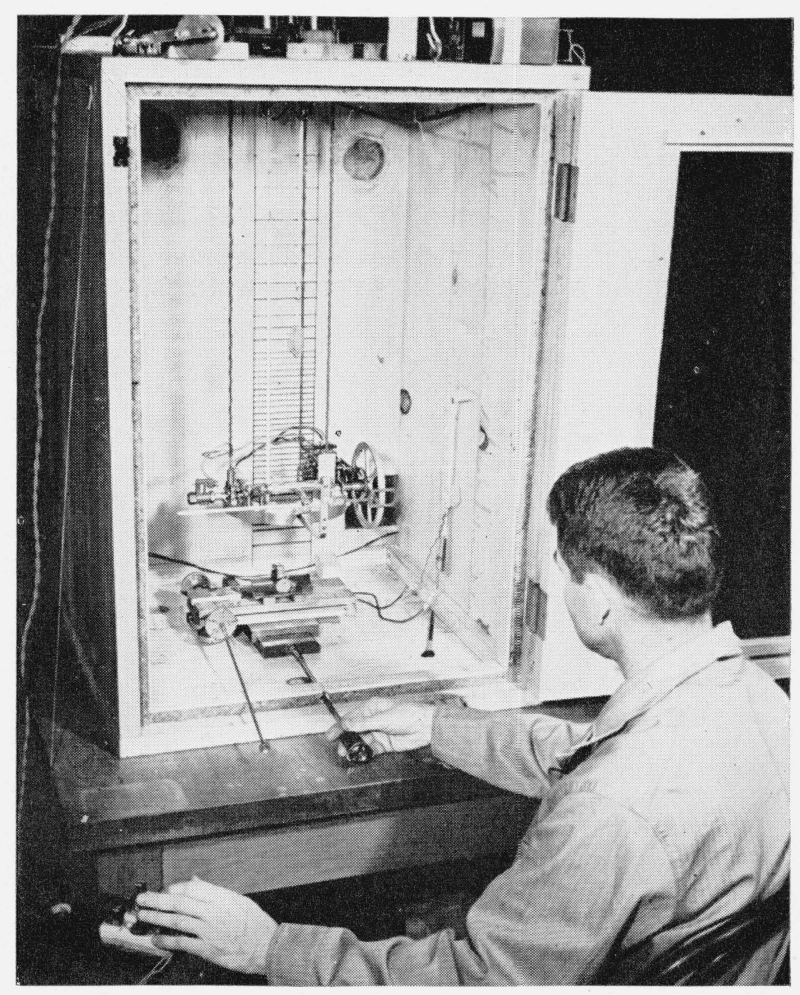

FIGURE 13. Temperature-controlled cabinet for coil measurement. 
TABLE 1. Coil measurements

[Values given are the average of all 9 wires]

(a) OVER-ALL WIDTH OF RECTANGULAR COIL AT $25^{\circ} \mathrm{C}$

\begin{tabular}{|c|c|c|c|}
\hline $\begin{array}{l}\text { Vertical posi- } \\
\text { tion, } y, \\
\text { relative to } \\
\text { bottom wires }\end{array}$ & Width & $\begin{array}{l}\text { Vertical posi- } \\
\text { tion, } y \text {, } \\
\text { relative to } \\
\text { bottom wires }\end{array}$ & Width \\
\hline $\mathrm{cm}$ & $\mathrm{mm}$ & $\mathrm{cm}$ & $\mathrm{mm}$ \\
\hline 0 & 80.5624 & 16.6 & 100.5781 \\
\hline 1.0 & 100. 2437 & 17.6 & .5784 \\
\hline 1. 4 & .5051 & 18.6 & .5786 \\
\hline 2. 0 & .7171 & 19.6 & .5800 \\
\hline 3. 0 & .6446 & 20.6 & .5799 \\
\hline 4. 0 & .6236 & 21.6 & .5807 \\
\hline 5.0 & .5880 & 22.6 & .5811 \\
\hline 6.0 & .5813 & 23. 6 & .5812 \\
\hline 7. 6 & .5739 & 24. 6 & .5820 \\
\hline 8. 6 & .5741 & 25.6 & .5815 \\
\hline 8. 9 & .5722 & 26.6 & .5831 \\
\hline 9.5 & .5719 & 27. 6 & .5829 \\
\hline 9.6 & .5729 & 28.6 & .5818 \\
\hline 9. 9 & .5714 & 29.6 & .5830 \\
\hline 10.5 & .5697 & 30.6 & .5837 \\
\hline 10.6 & .5711 & 31.6 & .5835 \\
\hline 10. 9 & .5699 & 32.6 & .5828 \\
\hline 11.5 & .5695 & 33.6 & .5829 \\
\hline 11. 6 & .5708 & 34.6 & .5828 \\
\hline 11.8 & .5703 & 35.6 & .5833 \\
\hline 12.5 & .5711 & 36.6 & .5834 \\
\hline 12.6 & .5716 & 37.8 & .5843 \\
\hline 12.9 & .5714 & 38.6 & .5849 \\
\hline 13.5 & .5722 & 40.1 & .5859 \\
\hline 13.6 & .5741 & 42.1 & .5869 \\
\hline 13.8 & .5734 & 44. 6 & .5875 \\
\hline 14.5 & .5753 & 47.1 & .5896 \\
\hline 14. 6 & .5757 & 49.8 & .5899 \\
\hline 14.8 & .5763 & 52.8 & .5911 \\
\hline \multirow[t]{2}{*}{15.6} & 100.5757 & 56.3 & .5906 \\
\hline & & 64.1 & 100.5869 \\
\hline
\end{tabular}

(b) Diameter OF WIRE

[Measured in direction of plane of coil]

\begin{tabular}{|c|c|c|c|c|c|c|c|}
\hline \multirow{2}{*}{ Wire No. } & \multicolumn{7}{|c|}{ Vertical distance from bottom wires } \\
\hline & $7.6 \mathrm{~cm}$ & $9.6 \mathrm{~cm}$ & $10.6 \mathrm{~cm}$ & $11.6 \mathrm{~cm}$ & $12.6 \mathrm{~cm}$ & $15.6 \mathrm{~cm}$ & Average \\
\hline & $\mathrm{mm}$ & $\mathrm{mm}$ & $\mathrm{mm}$ & $\mathrm{mm}$ & $m m$ & $\mathrm{~mm}$ & $\mathrm{~mm}$ \\
\hline $1 R_{--}$ & 0. 5665 & 0.5667 & 0.5680 & 0.5680 & 0.5672 & 0.5675 & 0.5673 \\
\hline $1 \mathrm{~L}$ & .5669 & .5669 & .5671 & .5664 & .5664 & .5662 & .5667 \\
\hline $2 \mathrm{R}_{--}$ & .5632 & .5633 & .5634 & .5634 & .5635 & .5635 & .5634 \\
\hline $2 \mathrm{~L}$ - & .5682 & .5684 & .5683 & .5683 & .5682 & .5679 & .5682 \\
\hline $3 \mathrm{R}_{-.}$ & .5644 & .5650 & .5649 & .5645 & .5652 & .5655 & .5649 \\
\hline $3 \mathrm{~L}$ & .5632 & .5632 & .5631 & .5623 & .5622 & .5619 & .5627 \\
\hline $4 \mathrm{R}_{-}$ & .5612 & .5617 & .5614 & .5612 & .5620 & .5614 & .5615 \\
\hline $4 \mathrm{~L}$ & .5613 & .5613 & .5612 & .5612 & .5612 & .5612 & .5612 \\
\hline $5 \mathrm{R}$. & .5602 & .5603 & .5599 & .5602 & .5599 & .5600 & .5601 \\
\hline $5 \mathrm{~L}_{-}$ & .5595 & .5600 & .5595 & .5601 & .5601 & .5600 & .5599 \\
\hline $6 \mathrm{R}$. & .5600 & .5602 & .5601 & .5601 & .5602 & .5602 & .5601 \\
\hline $6 \mathrm{~L} \ldots$ & .5600 & .5602 & .5601 & .5600 & .5601 & .5600 & .5601 \\
\hline $7 \mathrm{R}$ & .5602 & .5600 & .5601 & .5601 & .5602 & .5601 & .5601 \\
\hline $7 \mathrm{~L}_{-}$ & .5602 & .5602 & .5602 & .5602 & .5602 & .5602 & .5602 \\
\hline $8 R_{-}$ & .5602 & .5602 & .5602 & .5602 & .5602 & .5604 & .5602 \\
\hline $8 \mathrm{~L}_{-}$ & .5612 & .5615 & .5613 & .5615 & .5614 & .5617 & .5614 \\
\hline $9 \mathrm{R}_{\ldots}$ & .5630 & .5642 & .5638 & .5642 & .5644 & .5652 & .5641 \\
\hline $9 \mathrm{~L}_{\ldots}$ & .5604 & .5602 & .5603 & .5602 & .5603 & .5602 & .5603 \\
\hline A verage & 0.5622 & 0.5624 & 0.5624 & 0.5623 & 0.5624 & 0.5624 & 0.5624 \\
\hline
\end{tabular}

ments in the range 0 to $7.6 \mathrm{~cm}$ were made with less accuracy than those above $7.6 \mathrm{~cm}$. The field is so nearly uniform over this part of the coil that the error committed on this account is of no consequence. Between $7.6 \mathrm{~cm}$ and $15.6 \mathrm{~cm}$, measurements were made with the greatest care. Beginning at $7.0 \mathrm{~cm}$ the silk ties were spaced 1.0 $\mathrm{cm}$ on centers. Entries in table 1, a, corresponding to more than one vertical distance between consecutive ties $(9.5,9.6$, and $9.9 \mathrm{~cm}$, for example), give an idea of the bulging of the wire.

The method of measuring the diameter of the wire was similar to that used on the over-all width of the coil. The wire was hung vertically from a rotatable head and was loaded with a weight equal to the winding tension. Measurements were made on the wire by comparison with a gage block of the same nominal size by means of a precision micrometer. The micrometer could be raised relative to the wire for measurements in different positions along the sample. An attempt to obtain the diameter of the wire parallel to the plane of the coil from measurements on samples taken from the ends of the windings resulted in an uncertainty of $\pm 0.0004 \mathrm{~cm}$ on account of lack of roundness in the wire. This uncertainty was removed later by measurements made on samples cut from both sides of each turn of the winding. It was found possible to mark the orientation of each sample before it was cut from its respective turn and to retain this marking during the diameter measurement. Results of these measurements are shown in table $1, \mathrm{~b}$. As it turned out, the mean diameter of the wire parallel to the plane of the coil obtained in this way differed from the mean of all diameters obtained from the end samples by only $0.5 \mu$.

\section{The Distributed Field of the Electro- magnet}

The necessity of a knowledge of the normal component of the field over the region the coil occupies has been pointed out. This is not needed with much accuracy except over the lower part of the coil where the width changes rapidly. As previously described, the pole faces of the electromagnet were shimmed by trial with thin sheets of nickel to give essential uniformity over the bottom wires. The region over the bottom wires and up to a vertical distance of $7.0 \mathrm{~cm}$ was explored with a second movable proton resonance head, the first 
head being used to hold the field constant at a fixed point. Above $7.0 \mathrm{~cm}$ the field was not sufficiently uniform to get a recognizable resonance pip. The region above $7.0 \mathrm{~cm}$ was mapped with a device consisting of a small coil rotated by the shaft of a synchronous motor at 1,800 rpm. The emf generated in the coil at the various vertical positions was commutated and measured on a potentiometer. Significant differences from the value at the bottom wires of the coil began at about $5.0 \mathrm{~cm}$. The value of the field at the upper wires was about 3 gausses; this was neutralized to \pm 0.02 gauss by means of Helmholtz coils. Indication of neutrality was by a delicately suspended magnet in the neighborhood of the wires. The values of the field at various vertical positions relative to the

TABLE 2. Field $B(x, y)$ as a function of vertical distance

[ Values given for $B(x, y) / B_{r}$ have been averaged over $x$ at the given value of $y$ ]

\begin{tabular}{|c|c|c|c|}
\hline $\begin{array}{l}\text { Vertical posi- } \\
\text { tion, } y \text {, rela- } \\
\text { tive to bot- } \\
\text { tom wires }\end{array}$ & $\begin{array}{l}\text { Magnetic } \\
\text { field } \\
B(x, y) / B_{r}\end{array}$ & $\begin{array}{l}\text { Vertical posi- } \\
\text { tion, } y \text {, rela- } \\
\text { tive to bot- } \\
\text { tom wires }\end{array}$ & $\begin{array}{l}\text { Magnetic } \\
\text { field } \\
B(x, y) / B_{r}\end{array}$ \\
\hline $\mathrm{cm}$ & & $\mathrm{cm}$ & \\
\hline 0 & 1.0000 & 17.6 & 0.19 \\
\hline 1.0 & 0.99990 & 18.6 & .15 \\
\hline 1.4 & .99985 & 19.6 & .13 \\
\hline 2. 0 & .99980 & 20.6 & .12 \\
\hline 3.0 & .9995 & 21.6 & .10 \\
\hline 4. 0 & .9991 & 22.6 & .09 \\
\hline 5.0 & .9983 & 23.6 & .07 \\
\hline 6.0 & .996 & 24.6 & .06 \\
\hline 7.6 & .990 & 25.6 & .06 \\
\hline 8.6 & .972 & 26.6 & .05 \\
\hline \multirow[t]{2}{*}{8.9} & .958 & & \\
\hline & & 27.6 & .04 \\
\hline 9.5 & .928 & 28.6 & .04 \\
\hline 9.6 & .922 & 29.6 & .03 \\
\hline 9.9 & .897 & 30.6 & .03 \\
\hline 10.5 & .835 & 31.6 & .03 \\
\hline 10.6 & .822 & 32.6 & .02 \\
\hline 10.9 & .784 & 33.6 & .02 \\
\hline 11.5 & .693 & 34.6 & .02 \\
\hline 11.6 & .679 & 35.6 & .02 \\
\hline 11.8 & .646 & 36.6 & 01 \\
\hline \multirow[t]{2}{*}{12.5} & .546 & & \\
\hline & & 37.8 & .01 \\
\hline 12.6 & .535 & 38.6 & .01 \\
\hline 12.9 & .497 & 40.1 & .01 \\
\hline 13.5 & .431 & 42.1 & .01 \\
\hline 13.6 & .423 & 44.6 & .005 \\
\hline 13.8 & .403 & 47.1 & .005 \\
\hline 14.5 & .344 & 49.8 & .005 \\
\hline 14.6 & .337 & 52.8 & .004 \\
\hline 14.8 & .323 & 56.3 & .002 \\
\hline 15.6 & .272 & 64.1 & .001 \\
\hline 16.6 & .22 & & \\
\hline
\end{tabular}

value $B_{r}$ at some reference point within the magnet gap are shown in table 2 .

\section{$X$. Evaluation of the Factor $X$}

The factor $X$ has been defined as the effective width of the coil. In terms of the reference value $B_{r}$ used in table 2, eq 3 may be rewritten $X=$ $\left(B_{\tau} / \bar{B}\right) \mathcal{S}\left[B(x, y) / B_{r}\right] d x$. The above integral is evaluated numerically, as illustrated in table 3 , based on the data in tables 1 and 2 . The reference point was so chosen that within the experimental accuracy $B_{r}=\bar{B}$. From $y=0$ to $y=7 \mathrm{~cm}$ this could be determined with the resonance head; for the points beyond $7 \mathrm{~cm}$, the reference point was obtained by locating the rotating coil at the normal position of the "force conductors" of the rectangular coil. It should be pointed out that the value of the field indicated in table 3 is the mean value taken over the interval $\Delta y$. As it affects the final result, the uncertainty in the evaluation of $X$ can be properly divided into three parts having the following outer limits of error: width measurement $10 \mathrm{ppm}$, calibration of length standard $5 \mathrm{ppm}$, and field distribution $10 \mathrm{ppm}$.

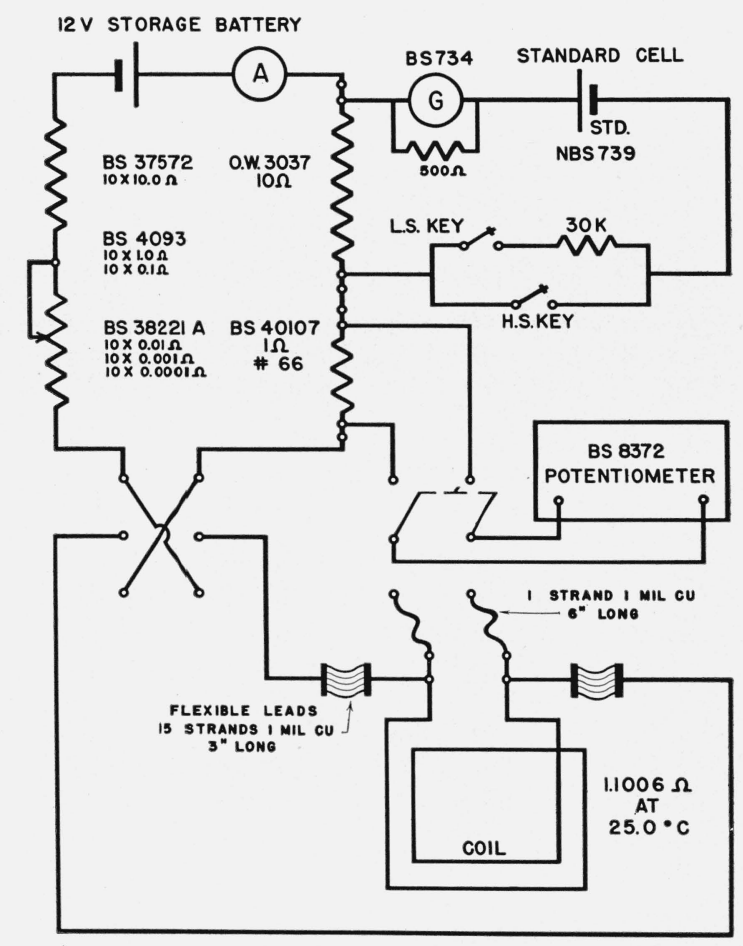

FiguRe 14. Electrical connections of magnetic balance. 
TABLE 3. Evaluation of the geometrical-factor $X$

[Values given for $B(x, y) / B_{r}$ from table 2 have been averaged over the interval $\Delta y$. Width given is the over-all width minus the mean wire diameter]

\begin{tabular}{|c|c|c|c|c|c|c|c|c|c|}
\hline$y$ & Width & $\Delta x$ & $\frac{B(x, y)}{B_{r}}$ & $\frac{B(x, y)}{B_{r}} \Delta x$ & $y$ & Width & $\Delta x$ & $\frac{B(x, y)}{B_{r}}$ & $\frac{B(x, y)}{B_{r}} \Delta x$ \\
\hline $\mathrm{cm}$ & $\mathrm{cm}$ & $\mathrm{cm}$ & & & $\mathrm{cm}$ & $\mathrm{cm}$ & $\mathrm{cm}$ & & \\
\hline 0 & 8.00000 & +8.00000 & 1.00000 & +8.00000 & 17.6 & 10.00160 & +0.00003 & 0.20 & +0.00001 \\
\hline 1.0 & 9.96813 & +1.96813 & 0.99995 & +1.96803 & 18.6 & 10.00162 & +.00002 & .17 & +.00000 \\
\hline 1.4 & 9.99427 & +0.02614 & .99988 & +0.02614 & 19. 6 & 10.00176 & +.00014 & .14 & +.00002 \\
\hline 2.0 & 10.01547 & +.02120 & .99983 & +.02120 & 20.6 & 10.00175 & -.00001 & .12 & -.00000 \\
\hline 3.0 & 10. 00822 & -.00725 & .99965 & -.00725 & 21.6 & 10.00183 & +.00008 & .11 & +.00001 \\
\hline 4.0 & 10. 00612 & -.00210 & .9993 & -.00210 & 22.6 & 10.00187 & +.00004 & .10 & +.00000 \\
\hline 5. 0 & 10. 00256 & -.00356 & .9987 & -.00356 & 23.6 & 10.00188 & +.00001 & .08 & +.00000 \\
\hline 6.0 & 10. 00189 & -.00067 & .9972 & -.00067 & 24.6 & 10.00196 & +.00008 & .06 & +.00000 \\
\hline 7.6 & 10. 00115 & -.00074 & .993 & -.00073 & 25.6 & 10.00191 & -.00005 & .06 & -.00000 \\
\hline 8.6 & 10.00117 & +.00002 & .983 & +.00002 & 26.6 & 10.00207 & +.00016 & .06 & +.00001 \\
\hline 8.9 & 10. 00098 & -.00019 & .96 & -.00018 & & & & & \\
\hline & & & & & 27.6 & 10.00205 & -.00002 & .05 & -.00000 \\
\hline & & & & & 28.6 & 10. 00194 & -.00011 & .04 & -.00000 \\
\hline 9.5 & 10.00095 & -.00003 & .94 & -.00003 & 29.6 & 10.00206 & +.00012 & .04 & +.00001 \\
\hline 9.6 & 10.00105 & +.00010 & .93 & +.00009 & 30.6 & 10.00213 & +.00007 & .03 & +.00000 \\
\hline 9.9 & 10.00090 & -.00015 & .91 & -.00014 & 31.6 & 10.00211 & -.00002 & .03 & -.00000 \\
\hline 10.5 & 10. 00073 & -.00017 & .87 & -.00015 & 32.6 & 10.00204 & -.00007 & .03 & -.00000 \\
\hline 10.6 & 10. 00087 & +.00014 & .83 & +.00012 & 33.6 & 10.00205 & +.00001 & .02 & +.00000 \\
\hline 10.9 & 10.00075 & -.00012 & .81 & -.00010 & 34.6 & 10.00204 & -.00001 & .02 & -.00000 \\
\hline 11.5 & 10.00071 & -.00004 & .74 & -.00003 & 35.6 & 10.00209 & +.00005 & .02 & +.00000 \\
\hline 11.6 & 10. 00084 & +.00013 & .69 & +.00009 & 36.6 & 10. 00210 & +.00001 & .02 & +.00000 \\
\hline 11.8 & 10.00079 & -.00005 & .66 & -.00003 & & & & & \\
\hline 12.5 & 10.00087 & +.00008 & .60 & +.00005 & 37.8 & 10.00219 & +.00009 & .01 & +.00000 \\
\hline & & & & & 38.6 & 10.00225 & +.00006 & .01 & +.00000 \\
\hline & & & & & 40.1 & 10.00235 & +.00010 & .01 & +.00000 \\
\hline 12.6 & 10.00092 & +.00005 & .54 & +.00003 & 42.1 & 10.00245 & +.00010 & .01 & +.00000 \\
\hline 12. 9 & 10.00090 & -.00002 & .52 & -.00001 & 44.6 & 10.00251 & +.00006 & .008 & +.00000 \\
\hline 13.5 & 10. 00098 & +.00008 & .46 & +.00004 & 47.1 & 10.00272 & +.00021 & .005 & +.00000 \\
\hline 13. 6 & 10.00117 & +.00019 & .43 & +.00008 & 49.8 & 10.00275 & +.00003 & .005 & +.00000 \\
\hline 13.8 & 10.00110 & -.00007 & .41 & -.00003 & 52.8 & 10.00287 & +.00012 & .004 & +.00000 \\
\hline 14.5 & 10.00129 & +.00019 & .37 & +.00007 & 56.3 & 10.00282 & -.00005 & .003 & -.00000 \\
\hline 14.6 & 10. 00133 & +.00004 & .34 & +.00001 & 64.1 & 10.00245 & -.00037 & .001 & -.00000 \\
\hline 14.8 & 10. 00139 & +.00006 & .33 & +.00002 & & & & & \\
\hline 15.6 & 10.00133 & -.00006 & .29 & -.00002 & \multirow{2}{*}{\multicolumn{5}{|c|}{$\frac{1}{B_{r}} B(x, y) \Delta x=10.00108$}} \\
\hline 16.6 & 10.00157 & +.00024 & .25 & +.00006 & & & & & \\
\hline
\end{tabular}

\section{Electrical Measurements}

The electrical circuit of the magnetic balance is shown in figure 14 . The current was held constant to about $1 \mathrm{ppm}$ by manual adjustment of the series resistance to keep the potential drop produced in the 10-ohm standard equal to the emf of a standard cell, as indicated by galvanometer $G$.

The standard resistor was submerged in a thermostated and stirred oil bath. The standard cell was maintained at a constant temperature in another thermostated bath. On disconnecting the storage battery and standard cell and closing the galvanometer circuit, no thermal emf was found of any appreciable magnitude. The error in the value of the current on account of the standards was not more than $3 \mathrm{ppm}$.

The resistance of the coil while carrying current was ascertained by comparing the potential across it with that across a standard resistance of $1 \mathrm{ohm}$ carrying the same current as shown in figure 14 .
In this way it was easy to find the resistance of the coil to $0.0001 \mathrm{ohm}$, allowing its temperature to be estimated to $0.1 \mathrm{deg} \mathrm{C}$. The resistance of the coil at a known temperature was measured by means of a Mueller bridge at the time it was mounted in the temperature-controlled cabinet for the width measurement. This resistance was 1.1006 ohms at $25^{\circ} \mathrm{C}$.

\section{Measurement of the Force}

The force produced on the current-carrying"coil was evaluated by comparison with the action of gravity upon a known mass that was placed upon the scalepan of the balance when the current was reversed. In case the comparison was not exact, a small correction was made to the mass in terms of the sensitivity of the balance, $s$, and the change in rest point of the balance, $\delta$. Reversing the current in the coil has several advantages: it keeps the heating of the coil constant, thereby helping 
to maintain the dead weight of the coil constant; it corrects for any lateral shift in the current; it corrects for attractive effects of the coil or other parts attached to the movable parts of the balance; and it doubles the observed force. Remembering the reversal of current and denoting the mass of the removable weight by $m$, and the acceleration of gravity by $g$, a working formula for the field $\bar{B}$ is given by

$$
\bar{B}=\frac{5(m+s \delta) g}{n I X[1+a(t-25)]},
$$

where $a$ is the coefficient of thermal expansion of the coil, and $t$ is its temperature. In practice the current $I$ has some fixed value according to the standards selected, and the magnetic field has some value consistent with the radio frequency applied to the proton probe to produce resonance; therefore, the mass, $m$, had to be adjusted by trial to a point where the correction, $s \delta$, was so small that the uncertainty in the sensitivity of the balance did not cause more than a tolerable error in the force. This mass, which is in the form of a cylinder, was then turned over to the Mass Section of the Bureau for standardization. A correction was made for the weight of displaced air to get the effective weight of the cylinder.

Turning points of the 2-kg Rueprecht balance were indicated by a spot of light reflected onto a glass scale from a mirror on the balance beam. The effective pointer length was $5.0 \mathrm{~m}$. The dead load on the balance was $1,500 \mathrm{~g}$. The change in weight on reversing the current was about $9.0 \mathrm{~g}$. The sensitivity of the system was such that $1 \mathrm{~mm}$ on the scale corresponded to 1 part in 60,000 of the change in force. The turning points were estimated to $1 / 10 \mathrm{~mm}$. The rest point, as estimated from averaging five turning points on one side and four on the other, would ordinarily be accurate to less than $1 / 10$ $\mathrm{mm}$; in this application there were fluctuations in the swings of the balance caused by varying air currents and building vibration, and perhaps slight variations in the average magnetic field that increase the uncertainty of a given rest point to several tenths of a millimeter. By taking the rest point about 10 times in a given run with the weight alternately off and on the scalepan, the fluctuations are averaged out giving the mean value of the difference in rest points, $\delta$, accurate to about $5 / 10 \mathrm{~mm}$.

\section{Gyromagnetic Ratio of the Proton}

The results of the measurements through January 7, 1949, are summarized in table 4 . Many spot checks were made during these runs, which indicated that the field distribution was remaining remarkably constant. Subsequently, when the field was shimmed symmetrically with thinner shim stock, measurable variations in the distribution with time were noted. Therefore, in taking the data of table 5 with symmetrical shims, the field distribution was plotted immediately before and after the measurement of $\bar{B}$ with the balance. Likewise, a new mass constructed of platinum-iridium was used on the balance for these readings. These results, obtained about 5 months later, are practically identical with the earlier ones. Considering all the runs, the average deviation is approximately $10 \mathrm{ppm}$.

It will be noticed that the acceleration of gravity $g$ appears in eq 5. The uncertainty [13] in this is taken as $5 \mathrm{ppm}$. The acceleration of gravity also arises in the absolute determination of the ampere [14]. Thus the current may be written

$$
I=k \sqrt{g_{1}},
$$

where $k$ is an experimental constant known to 10 ppm, to which must be added an error of $3 \mathrm{ppm}$ arising in the comparison of the current used in this experiment with the standards, and $g_{1}$ is the value of the acceleration of gravity used in the measurement of the ampere. Substituting eq 6 into eq 5

$$
\bar{B}=\frac{5(m+s \delta) g}{n k g_{1}^{1 / 2} X[1+a(t-25)]} .
$$

Letting

$$
h=g / g_{1}
$$

represent the ratio of the acceleration of gravity at the location of this experiment to that used in the determination of the ampere, eq 7 becomes

$$
\bar{B}=\frac{5(m+s \delta) h g_{1}^{1 / 2}}{n k X[1+a(t-25)]} .
$$

The advantage in this approach is that the quan tity $h g_{1}^{1 / 2}$ is known to approximately $2.5 \mathrm{ppm}$, whereas the acceleration of gravity is known only to $5 \mathrm{ppm}$. 
TABLE 4. Summary of results-first series

Acceleration of gravity at the balance $g$.

Geometrical factor of the coil (table 3)

Number of turns on coil $n$

$980.081 \mathrm{~cm} / \mathrm{sec}$

Expansion of the coil $a$ $10.00108 \mathrm{~cm}$ at $25^{\circ} \mathrm{C}$

Sensitivity of the balance $s$

$9.5 \times 10^{-6} / \mathrm{deg} \mathrm{C}$

$1.59 \mathrm{mg} / \mathrm{cm}$

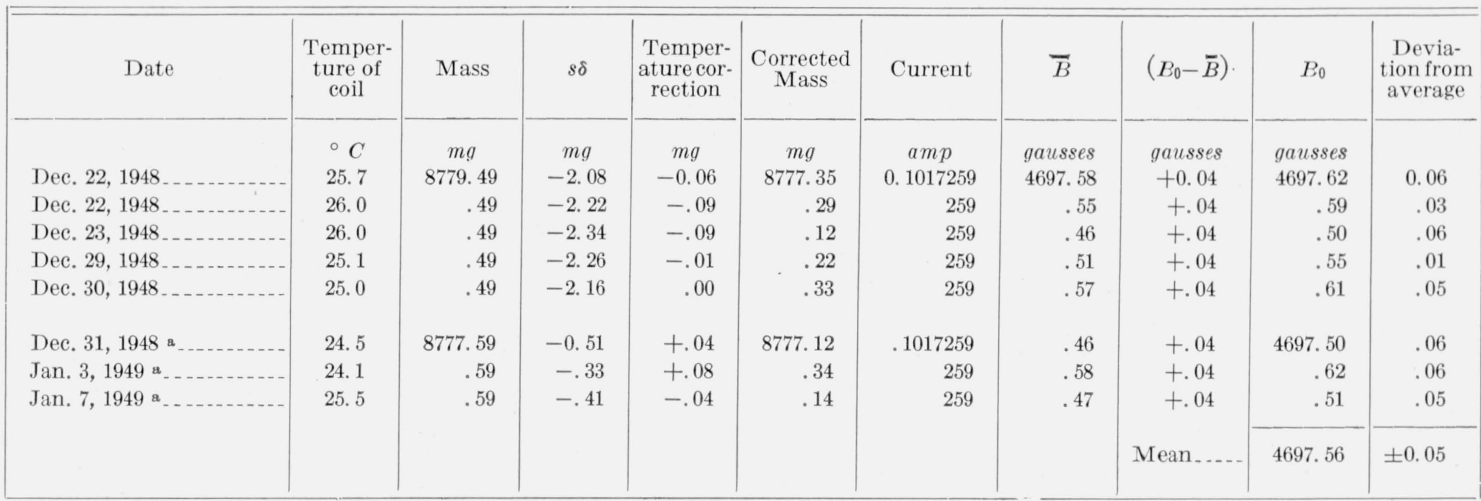

a New brass mass used in measurements.

$\nu=$ measured value of resonance frequency $=20,001,075 \pm 20 \mathrm{c} / \mathrm{s}$.

$\gamma_{p}=$ gyromagnetic ratio of proton $-2 \pi \nu / B_{0}=2.67523 \times 10^{4} \mathrm{sec}^{-1}$ gauss $^{-1}$.

TABLE 5. Summary of results-second series

In obtaining the data in this table, the following changes were made: A platinum-irridium mass was used $8777.29 \mathrm{mg}$.

Symmetrical shims were used, i. e., the same on each pole face.

The field distribution was checked before and after each run.

Current-0.1017274 ampere

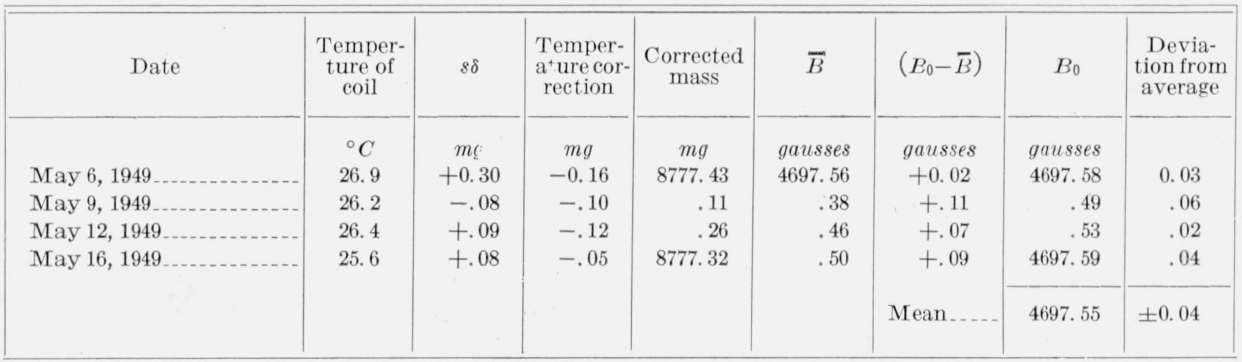

$\nu=20,001,075 \pm 20 \mathrm{c} / \mathrm{s}$.

$\gamma_{p}=$ gyromagnetic ratio of proton $=2 \pi \nu / B_{0}=2.67523 \times 10^{4} \mathrm{sec}^{-1}$ gauss $^{-1}$.

The value of the acceleration of gravity $g_{1}$ used in this experiment was $980.08 \mathrm{~cm} / \mathrm{sec}^{2}$, which was obtained along with $h$ from a gravity survey made at this Bureau in 1948 by the Geological Survey. This is slightly different from the value given by Curtis [15].

The known contributing errors listed in the tabulation below were estimated conservatively. The square root of the sum of their squares results in an uncertainty of $22 \mathrm{ppm}$ in the gyromagnetic ratio of the proton. The result can be stated as $\gamma_{p}=(2.67523 \pm 0.00006) \times 10^{4} \mathrm{sec}^{-1}$ gauss $^{-1}$ uncorrected for the diamagnetic effect of the orbital electron. The stated error is thought to be several times the corresponding probable error. If the diamagnetic correction [16], which was not included in the preliminary result [17], is applied to the above value one obtains $\gamma_{p}=(2.67528 \pm 0.00006)$ $\times 10^{4} \mathrm{sec}^{-1}$ gauss $^{-1}$.

The magnetic moment of the proton is known less accurately than the gyromagnetic ratio because of the uncertainty in the value of Planck's constant $h$. Using a value [18] of

$$
h=(6.6234 \pm 0.0011) \times 10^{-27} \mathrm{erg} \text { sec, }
$$

the magnetic moment of the proton is

$$
\mu_{p}=(1.4100 \pm 0.0002) \times 10^{-23} \text { dyne cm/gauss. }
$$




\begin{tabular}{|c|c|}
\hline Contributing errors & $\begin{array}{l}\text { Parts } \\
\text { per } \\
\text { million }\end{array}$ \\
\hline Platinum-iridium mass $m_{\ldots} \ldots$ & 1 \\
\hline Precision of balance used in this experiment & 9 \\
\hline Gravity $h g_{1} 1 / 2$ & 3 \\
\hline $\begin{array}{l}\text { Experimental constant in measurement of } \\
\text { the ampere in absolute units }\end{array}$ & 10 \\
\hline Comparison of current with standards & 3 \\
\hline Width of coil & 10 \\
\hline Length standard & 5 \\
\hline Field distribution & 10 \\
\hline Neutralization of stray field at upper wires_ & 5 \\
\hline $\begin{array}{l}\text { Adjustment of resonance pip to reference } \\
\text { point on oscilloscope }\end{array}$ & 4 \\
\hline $\begin{array}{l}\text { Calibration of Helmholtz coils used for field } \\
\text { distribution }\end{array}$ & 4 \\
\hline Resonance frequency & 1 \\
\hline Effect of ferric ions in sample & 4 \\
\hline
\end{tabular}

\section{Comparison With Other Measure- ments}

Gardner and Purcell [19] have just completed a measurement of the ratio of the precession frequency of the proton, $\omega=\gamma_{p} B_{0}$, to the cyclotron frequency, $\omega_{e}=e B_{0} / m$, of a free electron in the same magnetic field. After making the diamagnetic correction, they give for the ratio $\omega / \omega_{e}=$ $(1.52100 \pm 0.00002) \times 10^{-3}$. This ratio is the magnetic moment of the proton in Bohr magnetons and agrees very well with the value $\mu_{p}=(1.52106 \pm$ $0.00007) \times 10^{-3}$ Bohr magneton obtained by Taub and Kusch [20]. These values of $\mu_{p}$ may be combined with the value of the gyromagnetic ratio of the proton to give a value of $e / \mathrm{m}$. In this calculation it is preferable to use the value of Gardner and Purcell because of its greater accuracy and because the electron moment correction is not involved. This value of $e / m$ becomes $e / m=$ $\gamma_{p} \cdot \omega_{e} / \omega=(1.75890 \pm 0.00005) \times 10^{+7}$ emu gram $^{-1}$. This differs only slightly from the previously published value [21], which was obtained by combining our preliminary value of $\gamma_{p}$ and the measurement of Taub and Kusch. Figure 15 shows the new value of $e / m$ as compared with other experimental and recommended values summarized by DuMond and Cohen [18].

The value of the gyromagnetic ratio of the proton, and hence the above value of $e / m$, has recently received further confirmation since it has

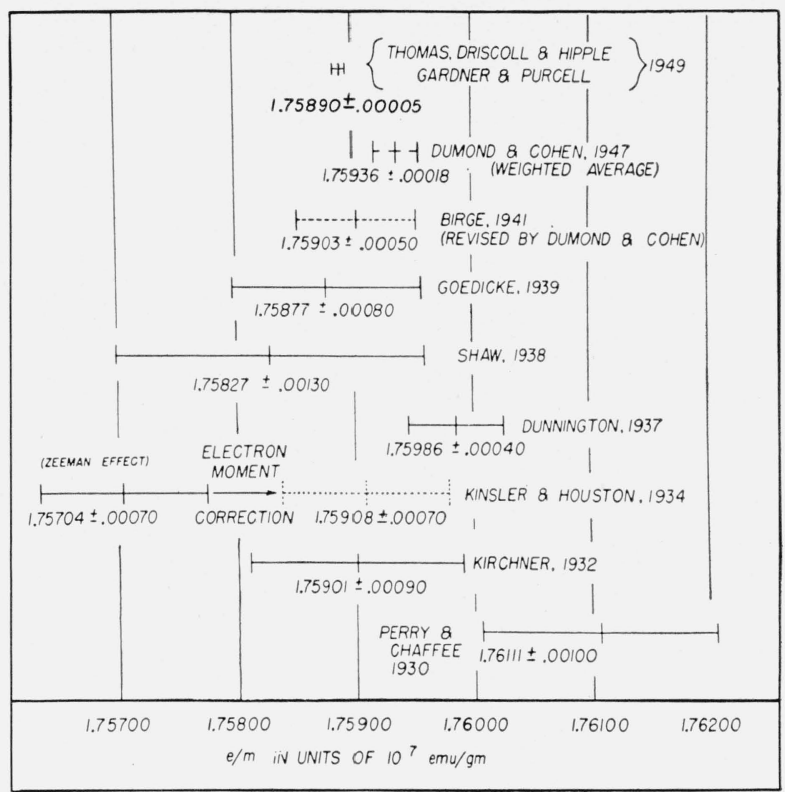

Figure 15. Values for e/m as summarized by DuMond and Cohen compared with the measurements reported in this paper.

been used in determining the Faraday [22] in which excellent agreement with the iodine voltameter was obtained.

The contributions of other staff members are gratefully acknowledged, and in particular the authors thark the following: Charles Moon, for advice on the construction and measurement of the coil; L. P. Slivka, for assistance in making many measurements on the coil; L. B. Macurdy, for the calibration of the masses; W. J. Hamer, for the calibration of standard cells; Anne YungKwai, for the calibration of standard resistors; and I. H. Fullmer, for the calibration of gage blocks. Helpful suggestions were received from F. B. Silsbee, Chester Snow, and R. D. Huntoon.

\section{References}

[1] I. I. Rabi, S. Millman, P. Kusch, and J. R. Zacharias, Phys. Rev. 55, 526 (1939).

[2] F. Bloch, Phys. Rev. J0, 460 (1946).

[3] E. M. Purcell, H. C. Torrey, and R. V. Pound, Phys. Rev. 69, 37 (1946).

[4] N. Bloembergen, E. M. Purcell, and R. V. Pound, Phys. Rev. 73, 679 (1948).

[5] L. M. Brown and E. M. Purcell, Phys. Rev. 75, 1262 (1949).

[6] M. H. Pellat, J. phys. 6, 175 (1887). 
[7] A. Cotton and G. Dupouy, Congr. intern. d'Electricite 3 Sect. $\boldsymbol{2}, 208$ (1932).

[8] F. A. Scott, Phys. Rev. 46, 633 (1934).

[9] G. H. Briggs and A. F. A. Harper, J. Sci. Instruments 13, 119 (1936).

[10] H. A. Thomas and R. D. Huntoon, Rev. Sci. Instruments 20, 516 (1949).

[11] H. L. Curtis, C. Moon, and C. M. Sparks, J. Research NBS 21, 375 (1938) RP1137.

[12] C. Moon, J. Research NBS 10, 249 (1933) RP528.

[13] H. L. Dryden, J. Research NBS 99, 303 (1942) RP1502.

[14] R. W. Curtis, R. L. Discoll, and C. L. Critchfield, J. Research NBS 28, 133 (1942) RP1449.

[15] H. L. Curtis, J. Research 33, NBS 235 (1944) RP1606.

[16] W. E. Lamb, Jr., Phys. Rev. 60, 817 (1941). (The correction referred to on p. 581 is for the hydrogen atom. N. F. Ramsey, Phys. Rev. 7y, 567 (1950) has calculated the correction for the $\mathrm{H}_{2}$ molecule to be $2.7 \times 10^{-5}$. If the frequency shift between $\mathrm{H}_{2}$ and the Bureau sample were determined, the result reported might be changed slightly).

[17] H. A. Thomas, R. L. Driscoll, and J. A. Hipple, Phys. Rev. 75, 902 (1949).

[18] J. W. M. DuMond and E. R. Cohen, Rev. Modern Phys. 20, 82 (1948).

[19] J. H. Gardner and E. M. Purcell, Phys. Rev. $\boldsymbol{8 6}$, $1262(1949)$

[20] H. Taub and P. Kusch, Phys. Rev. 75, 1481 (1949); Kusch and Taub, $\mathbf{7 5 ,} 1477$ (1949).

[21] H. A. Thomas, R. L. Driscoll, and J. A. Hipple, Phys. Rev. 75, 992 (1949).

[22] J. A. Hipple, H. Sommer, and H. A. Thomas, Phys Rev. \%6, 1877 (1949).

Washington, September 29, 1949. 